\title{
XXIII. Mittheilungen aus dem krystallographischen Laboratorium des „Central Technical College“.
}

\author{
Von
}

William J. Pope in London.

Nr. II. Diè Krystallformen der Natriumsalze der substituirten Anilsäuren.

(Mit 8 Textfiguren.)

Die nachstehend beschriebenen Salze sind sämmtlich von Herrn A. R. Ling dargestellt worden (Journ. Chem. Soc. 1892, 558). Es sind die Natriumsalze der Bromanil-, Chloranil- und Bromchloranilsäure, sowie eine Molekularverbindung oder Doppelsalz aus zwei Molekulen Natriumbromanilat und einem Molekul Natriumchloranilat.

Bromanilsaures Natrium, $\mathrm{C}_{6} \mathrm{Br}_{2}(\mathrm{ONa})_{2} \mathrm{O}_{2}, 4 \mathrm{H}_{2} \mathrm{O}$.

Die Krystalle bilden $6-8 \mathrm{~mm}$ lange und etwa $1 \mathrm{~mm}$ dicke Prismen von dunkler Farbe; sie sind undurchsichtig und zeigen erst in dunnen Splittern oder gepulvert eine blutrothe Farbe, schwachen Dichroismus und eine starke Doppelbrechung. Sie bilden meist wirre, von einem Punkte ausstrahlende Aggregate oder auch Parallelverwachsungen. Die vorherrschenden Formen $a\{100\}$ und $b\{010\}$ sind gekrümmt und daher schlecht messbar, die nur gering entwickelten $p\{\bar{T} 10\}$ und $c\{001\}$ sind dagegen gut spiegelnd. $r\{101\}$ ist von wechseln-

Fig. 1.

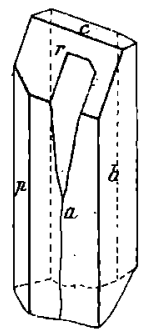

Fig. 2.

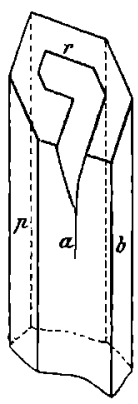

Fig. 3.

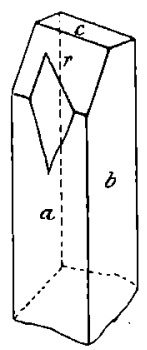

- der Grösse und beinabe stets durchbrochen von einem, in der Richtung der $c$-Axe den Krystall durchsetzenden Hohlraume, welcher die in Fig. 1, 2 und 3 (Rückansichten der Krystalle) 
angedeutete Form besitzt und der ausser $r$ auch öfters noch die Fläche $c\{001\}$ theilweise wegschneidet. $p\{110\}$ und $c\{001\}$ fehlen auch zuweilen. Eine Andeutung von Spaltbarkeit parallel $c\{001\}$ ist zu erkennen, doch ist der Bruch im Allgemeinen sehr unregelmässig. Zwillingsbildung nach $p\{110\}$ gewöhnlich. . Die häufigsten Gestalten sind in Fig. 1, 2 und 3 wiedergegeben.

Krystallsystem: Triklin.

$$
\begin{gathered}
a: b: c=0,8768: 1: 0,8100 \\
\alpha=69^{\circ} 28^{\prime}, \quad \beta=87056^{\prime}, \quad \gamma=71^{\circ} 49^{\prime} .
\end{gathered}
$$

Beobachtele Formen: $a\{100\}, b\{010\}, c\{001\}, p\{1 T 0\}, r\{101\}$.

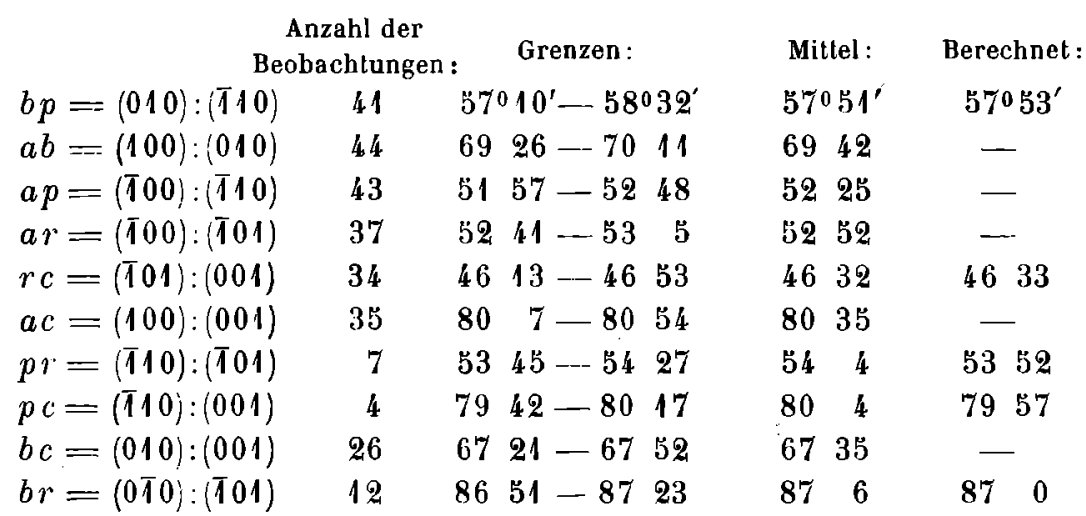

Chloranilsaures Natrium, $\mathrm{C}_{6} \mathrm{Cl}_{2}\left(\mathrm{ONa}_{2} \mathrm{O}_{2} \cdot 4 \mathrm{H}_{2} \mathrm{O}\right.$.

Die Substanz erscheint aus heisser wăsseriger. Lösung in sehr langen, dunkel gefărbten Prismen, ähnlich den vorbergehend beschriebenen, jedoch grösser als diese; die Formen $a\{100\}$ und $b\{010\}$ sind noch mehr gewölbt

Fig. 1.

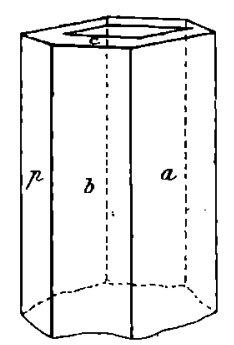
als bei jenen. Isolirte Krystalle sind nur spärlich anzutreffen wegen der grossen Neigung der Substanz zur Bildung von Aggregaten und Parallelverwachsungen, deren Enden in sehr lange und feine, durchsichtige Nadeln auslaufen, welche eine starke Doppelbrechung, schwachen Dichroismus und blutrothe Farbe zeigen. Die Fläche $c\{001\}$ ist stets durch eine Aushöhlung wie in Fig. 4 abgebildel durchbrochen, welche bis zu etwa $2 \mathrm{~mm}$ Tiefe in der Richtung der $c$-Axe in den Krystall hineingeht. Die Form $c\{001\}$ ist selten und wenn vorhanden nur schmal, aber glänzend; die Reflexe derselben sind wegen der Aushöhlung schwach. Keine Zwillingsbildung wahrnehmbar, hingegen eine unvollkommene Spaltbarkeit parallel $c\{001\}$. Die Axenlange $c$ muss vorderband, aus Mangel an Formen, unbestimmt bleiben. 
Krystallsystem: Triklin.

$$
\begin{aligned}
a: b: c & =0,8743: 1: ? ; \\
\alpha=88^{\circ} 8^{\prime}, \quad \beta & =89^{\circ} 51^{\prime}, \quad \gamma=72^{0} 32^{\prime} .
\end{aligned}
$$

\begin{tabular}{|c|c|c|c|c|}
\hline & $\begin{array}{l}\text { ahl d } \\
\text { chtur }\end{array}$ & Grenzen & Mitlel: & Berechnet \\
\hline$b p=(010):($ T10 $)$ & 14 & $58^{0} 9^{\prime}-59^{0} 23^{\prime}$ & $59^{\circ} 11^{\prime}$ & $58^{0} 58^{\prime}$ \\
\hline$a p=(\bar{T} 00):(\bar{T} 10)$ & 17 & $48 \quad 12-48 \quad 47$ & $48 \cdot 33$ & $\ldots$ \\
\hline$a b=(100):(010)$ & 16 & $728-7253$ & 7229 & 一 \\
\hline$a c=(100):(001)$ & 18 & $8856-8928$ & $89 \quad 15$ & - \\
\hline$a c=(\bar{T} 00):(001)$ & 12 & $9021-9053$ & 9036 & $90 \quad 45$ \\
\hline$b c=(010):(001)$ & 21 & $87 \quad 29-88 \quad 18$ & 8759 & - \\
\hline$b c=(0 \pi 0):(001)$ & 7 & $9148-92 \quad 40$ & $92 \quad 2$ & 921 \\
\hline
\end{tabular}

Beobachtete Formen: $a\{100\}, b\{010\}, c\{001\}, p\{\overline{1} 10\}$.

\section{Bromchloranilsaures Natrium, $\mathrm{C}_{6} \mathrm{Br} \mathrm{Cl}(\mathrm{ONa})_{2} \mathrm{O}_{2} \cdot 4 \mathrm{H}_{2} \mathrm{O}$.}

Die Beschaffenheit dieser Krystalle ist eine sehr unzulängliche und deshalb sind die unten gegebenen Werthe nur als angenäherle zu betrachten. Die Krystalle bilden dưnne, gedrehte Prismen von dunkler Farbe, welche im Pulver starke Doppelbrechung und schwachen Dichroismus, wie die beiden vorigen, erkennen lassen. $a\{100\}$ und $b\{010\}$ sind gekrümmt, und die Flächen $r\{\overline{101}\}$ und $c\{001\}$ erscheinen nur in schmalen Randleisten an den auch hier auftretenden Aushöhlungen wie in Fig. 5 ersichtlich. Die Krystalle sind ungemein spröde und zerbrechlich, nach $c\{001\}$ scheint eine sehr unvollkommene Spaltbarkeit vorhanden zu sein. $p\left\{T_{1} 0\right\}$ ist selten, aber stets glänzend und gut spiegelnd.

Krystallsystem: Triklin.

$$
\begin{gathered}
a: b: c=0,888: 1: 0,814 ; \\
\alpha=69^{\circ} 59^{\prime}, \quad \beta=8703^{\prime}, \quad \gamma=71058^{\prime} .
\end{gathered}
$$

Beobachtete Formen: $a\{100\}, b\{010\}, c\{001\}, p\{110\}, r\{101\}$.

Anzahl der

Beobachtungen : Grenzen :

$$
\begin{aligned}
& b p=(010):(\overline{1} 10) \\
& 18 \\
& a b=(100):(010) \\
& a p=(\bar{T} 00):\left(T_{1} 0\right) \\
& 17 \\
& 18 \\
& a r=(100):(\text { T01 }) \\
& r c=(\bar{T} 01):(001) \\
& a c=(100):(001) \\
& b c=(010):(001)
\end{aligned}
$$

52 $34-53 \quad 8$

Mittel: Berechnet:

$57029^{\prime}$

$57^{\circ} 33^{\prime}$

6936

5251

$53 \cdot 28$

4650

7951

$67 \quad 49$

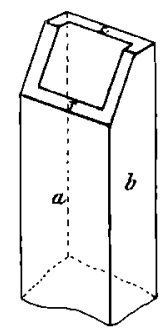




\section{Doppelsalz von chlor- und bromanilsaurem Natron, $2 \mathrm{C}_{6} \mathrm{Br}_{2}(\mathrm{ONa})_{2} \mathrm{O}_{2} \cdot 4 \mathrm{H}_{2} \mathrm{O}+\mathrm{C}_{6} \mathrm{Cl}_{2}(\mathrm{ONa})_{2} \mathrm{O}_{2} \cdot 4 \mathrm{H}_{2} \mathrm{O}$.}

Es wurden Krystalle, welche auf zweierlei Art dargestellt waren, untersucht. A. solche erbalten durch Einwirkúng von Aetznatron auf $p$-Dichlorodibromchinon und B. solche, die durch einfaches Zusammenkrystallisiren des Chlor- und Bromanilates aus Wasser erhalten worden waren. Beide erwiesen sich als identisch. Die Krystalle sind grosse, beinahe schwarze Prismen, sehr brüchig und nicht zu unterscheiden von denen des Bromanilates; erst die Messungen ergaben einige kleine Unterschiede. Die Krystalle zeigen die in Fig. 1, 2 und 3 angedeuteten Formen. Zwillingsbildung nach $p\{\overline{1} 10\}$ wie beim Bromanilat. $a\{100\}$ und $b\{010\}$ sind gekrummt, $p\{\pi 10\}$ schmal, bäufig fehlend, aber glänzend; auf $r\{101\}$ erscheinen ebenfalls die bei den beiden anderen Salzen erwähnten Höhlungen. Andeutung von Spaltbarkeit nach $c\{001\}$, welch letztere Fläche glänzend und gut spiegelnd ist. Das Krystallpulver lässt starke Doppelbrechung und schwachen Dichroismus erkennen, die Farbe ist weinroth.

Krystallsystem : Triklin.

Beobachtete Formen : $a\{100\}, b\{010\}, c\{001\}, p\{110\}, r\{\bar{T} 01\}$.

Krystalle A.

$$
a: b: c=0,8825: 1: 0,8163 \text {; }
$$

$$
\alpha=69^{\circ} 48^{\prime}, \quad \beta=87^{\circ} 14^{\prime}, \quad \gamma=72^{\circ} 11^{\prime} \text {. }
$$

Anzahl der

\begin{tabular}{|c|c|c|}
\hline$p=(010):(\bar{T} 10)$ & 35 & $57017^{\prime}-57046$ \\
\hline$a b=(100):(010)$ & 37 & $69 \quad 19-70 \quad 7$ \\
\hline$a p=(\bar{T} 00):(\bar{T} 10)$ & 35 & $52 \quad 16-5256$ \\
\hline$a r=(\bar{T} 00):(\bar{T} 01)$ & 28 & $53 \quad 3-53$ \\
\hline${ }^{\circ} c=(\bar{T} 01):(001)$ & 19 & $4629-4659$ \\
\hline$c c=(100):(001)$ & 21 & $7935-8037$ \\
\hline$r=(\bar{T} 10):(\bar{T} 01)$ & 7 & $5332-54$ \\
\hline$b c=(010):(001)$ & 17 & $6738-6755$ \\
\hline$b r=(0 \bar{T} 0\rangle:(\bar{T} 01)$ & 9 & $86 \quad 34-87$ \\
\hline$=(010):(\bar{T} 01)$ & 1 & - \\
\hline
\end{tabular}

$$
\text { Beobachtungen: Grenzen: }
$$

\begin{tabular}{|c|c|}
\hline Aittel: & Berechnet \\
\hline $57037^{\prime}$ & $57038^{\prime}$ \\
\hline $69 \quad 52$ & - \\
\hline 52 30 & - \\
\hline 536 & - \\
\hline $\begin{array}{lll}46 & 38\end{array}$ & $\begin{array}{ll}46 \quad 49\end{array}$ \\
\hline $80 \quad 5$ & - \\
\hline $53 \quad 57$ & $54 \quad 13$ \\
\hline $67 \quad 45$ & $\begin{array}{ll}66 & 58\end{array}$ \\
\hline $\begin{array}{ll}86 & 45\end{array}$ & - \\
\hline $93 \quad 23$ & 932 \\
\hline
\end{tabular}

Krystalle B.

$$
a: b: c=0,8825: 1: 0,8143 ;
$$

$$
\alpha=69^{\circ} 56^{\prime}, \quad \beta=87^{\circ} 7^{\prime}, \quad \gamma=72^{\circ} 11^{\prime} \text {. }
$$

Anzahl der

$$
\text { Beobachtungen : }
$$

Grenzen :

Mittel :

Berechnet:

$\begin{array}{llll}b p=(010):(T 10) & 39 & 57011^{\prime}-57054^{\prime} & 57039 \\ a b=(100):(010) & 37 & 6934-7011 & 6951 \\ a p=(\bar{T} 00):(\bar{T} 10) & 41 & 5223-5241 & 5228\end{array}$


Mittheilungen a. d. krystallogr. Lahorat. des »Central Technical College . II. 533

\begin{tabular}{|c|c|c|c|c|}
\hline \multicolumn{3}{|c|}{$\begin{array}{c}\text { Anzahl der } \\
\text { Beobachtungen }\end{array}$} & \multirow{2}{*}{$\begin{array}{l}\text { Mitlel: } \\
53^{0} 12\end{array}$} & \multirow{2}{*}{$\begin{array}{c}\text { Berechnet } \\
-\end{array}$} \\
\hline$a r=(T 00):(101)$ & 25 & $53^{0} 4^{\prime}-53^{0} 17^{\prime}$ & & \\
\hline$r c=(T 01):(001)$ & 14 & $4621-4659$ & $46 \quad 41$ & $46^{\circ} 4.8^{\prime}$ \\
\hline$a c=(100):(001)$ & 23 & $7936-80$ 25 & $80 \quad 0$ & - \\
\hline$p r=(\bar{T} 10):(\bar{T} 01)$ & 9 & $53 \quad 47-54 \quad 19$ & $54 \quad 2$ & 5421 \\
\hline$b c=(010):(001)$ & 18 & $67 \quad 4.1-68 \quad 7$ & 6751 & - \\
\hline$b r=(0 \pi 0):(T 01)$ & 4 & $\begin{array}{llll}86 & 28 & -87 & 12\end{array}$ & $86 \quad 41$ & 8658 \\
\hline
\end{tabular}

Als mittleres Axenverhältniss ergiebt sich :

$$
\begin{gathered}
a: b: c=0,8825: 1: 0,8153 ; \\
\alpha=69^{\circ} 52^{\prime}, \quad \beta=87^{\circ} 101^{\prime}, \quad \gamma=72^{\circ} 11^{\prime} .
\end{gathered}
$$

Infolge des gestörten Wachsthums der vorstehenden Salze besitzen die mitgetheilten Constanten nur einen geringen Grad von Genauigkeit, namentlich jene des Chlor- und des Bromochloranilates, welche nur wenige Messungen zuliessen. Die grosse Uebereinstimmung der Dimensionen des Bromanilates mit jenen des Doppelsalzes ist sehr bemerkenswerth und nicht oft beobachtet worden; im Mineralreiche zeigen eine ähnliche Uebereinstimmung Alstonit und Barytocölestin mit ibren resp. Componenten, obgleich Alstonit von manchen Mineralogen als isomorphe Mischung von Baryum- und Calciumcarbonat angesehen wird.

Es ist weiter erwähnenswerth, dass dieses Doppelsalz von der Formel $2 \mathrm{C}_{6} \mathrm{Br}_{2}(\mathrm{ONa})_{2} \mathrm{O}_{2} .4 \mathrm{H}_{2} \mathrm{O}+\mathrm{C}_{6} \mathrm{Cl}_{2}(\mathrm{ONa})_{2} \mathrm{O}_{2} \cdot 4 \mathrm{H}_{2} \mathrm{O}$ die einzige Verbindung ist, welche aus der wässerigen Lösung der einfachen Salze erhalten wurde. Herr Ling hat versucht, isomorphe Mischungen der beiden Salze zu erhalten, jedoch ohne Erfolg; es scheidet sich das Doppelsalz ab und die uberschiessenden Einzelbestandtbeile gelangen später zur Abscheidung.

Dieser Fall bietet also ein neues Beispiel für Substanzen, welche bei zweifellos grösster Analogie in chemischer und krystallographischer Beziehung nichtsdestoweniger nicht isomorph im gewöhnlichen Sinne sind, d. h. Natriumbromanilat und Natriumchloranilat besitzen nicht die Eigenschaft, in beliebiger Menge zusammenzukrystallisiren.

\section{Nr. III.}

\section{Die Krystallformen der stereoisomeren $\alpha \alpha^{\prime}$. Dimethylpimelinsäuren.}

Die gewöholichen physikalischen und chemischen Eigenschaften der beiden kürzlich beschriebenen (Kipp ing, Journ. Ghem. Soc. 1895, 139) stereoisomeren $\alpha \alpha^{\prime}$-Dimethylpimelinsäuren, nämlich der $p$-Dimethylpimelinsăure und der Antidimethylpimelinsäure, sind äusserst gleichartig, das elektrische Leitungsvermögen ist identisch und ibre Schmelzpunkte liegen sehr nahe bei einander. 
Dic vorliegende krystallographische Untersuchung nun hat weitere Beweise ergeben, dass die beiden Säuren total verschieden sind.

$$
\begin{aligned}
& \text { Para-Dimethylpimelinsäure, } \\
& \mathrm{COOH} . \mathrm{CH}\left(\mathrm{CH}_{3}\right) . \mathrm{CH}_{2} . \mathrm{CH}_{2} \cdot \mathrm{CH}_{2} . \mathrm{CH}\left(\mathrm{CH}_{3}\right) . \mathrm{COOH} \text {. }
\end{aligned}
$$

Schmelzpunkt 810-81;50. Dargestellt von Kipping (l. c.).

Dic Krystalle, welche sich aus kalter wässeriger Lösung dieser Säure ausscheiden, bilden weisse, opake Prismen von $4-5 \mathrm{~mm}$ Länge, welche an den Kanten und Ecken durchsichtig sind; die Undurchsichtigkeit der übrigen Masse wird durch Farnkraut-ăhnliche Einschlusse bedingt. Diese Krystalle zeigen meist nur $c\{001\}$ und $m\{110\}$, seltener noch $a\{100\}$ als ganz schmale Fläche. Bei der Verflüchtigung der Süure bilden sich nach

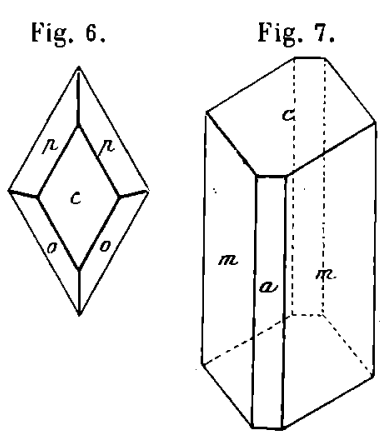
der $c$-Axe flache Tafeln, eine Ausbildungsweise der Krystalle, welche auch manchmal an den aus Wasser ausgeschiedenen beobachtet wird. Die Tafeln scheinen gebildet zu sein von den Formen $c\{001\}, o\{111\}$ und $p\{111\}$ (Fig. 6). Auf der Tafelfläche halbiren die Auslöschungsrichtungen die Winkel und eine optische Axe tritt beinabe senkrecht zu $c\{001\}$ und in der Ebene $b\{010\}$ gelegen aus. Doppelbrechung und Dispersion sind schwach. Die Krystalle sind indessen wegen allzu starker Krummung der Flächen zu Messungen ungeeignet. Ein wenig besser entwickelte, durchsichtige Krystalle von etwa $\frac{1}{2} \mathrm{~mm}$ Länge und $\frac{1}{5} \mathrm{~mm}$ Breite wurden erhalten beim Verdunsten einer wässerigen Lösung über Schwefelsäure. Dieselben haben eine rhombőderähnliche Form, gebildet von $c\{001\}$, $m\{110\}$ und zuweilen $a\{100\}$, Fig. 7, ein einziger Krystall zeigte auch eine Fläche von $o\{11 \overline{1}\}$. Die Krystalle gehören dem monoklinen Systeme an; die Flächen sind aber alle so gekrümmt, dass sie.keine genaueren Messungen erlauben. Eine charakteristische Spaltbarkeit ist nicht vorhanden. Der Brechungsindex ist ungefähr derselbe wie jener des Cederholzöles. Die Krystalle sind von derselben Art wie die oben beschriebenen, die optische Axe ist durch $c$ sichtbar.

Krystallsystem : Monoklin.

\begin{tabular}{|c|c|c|c|c|}
\hline & $\begin{array}{l}\text { ahl der } \\
\text { chtungen }\end{array}$ & Grenzen : & Miltel : & Berechnet \\
\hline$m=(110):(1 \bar{T} 0)$ & 21 & $80^{\circ} 6^{\prime}-83^{0} 50^{\prime}$ & $81^{\circ} 53^{\prime}$ & - \\
\hline$m m=(110):(T+0)$ & 18 & $95 \quad 10-100 \quad 48$ & 9832 & $98^{\circ} 7^{\prime}$ \\
\hline$m c=(110):(001)$ & 15 & $5448-5824$ & $56 \quad 42$ & - \\
\hline
\end{tabular}

$$
a: b: c=1,263: 1: 1,062 ; \beta=43^{0} 23^{\prime} .
$$


Mittheilungen a. d. klystallogr. Laborat. des "Central 'Technical Collegeu. III. $\mathbf{5 3 5}$

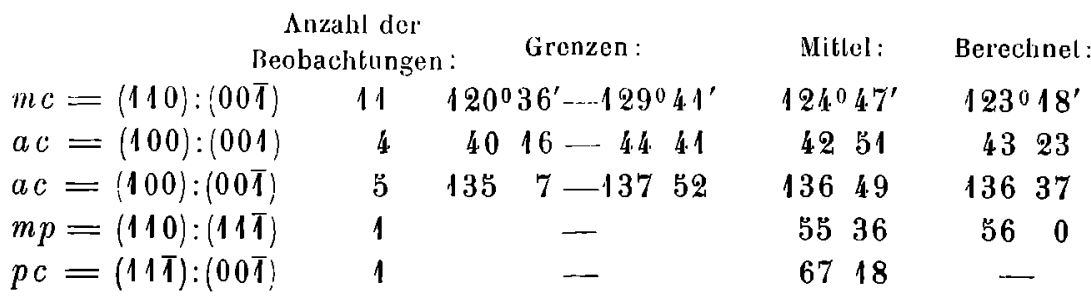

Bringt man die Substanz auf einem Objectglase zum Schmelzen und bedeckt mit einem Deckgläschen, so krystallisirt die Säure leicht von verschiedenen Centren aus, das Product ist durchsichtig und zeigt zuweilen eine optische Axe. Längs den Beriuhrungslinien der zahlreichen Individuen sind feine Bläschen zu erkennen, durch welche Eigenthümlichkeit die Substanz erkannt werden kann. Die Krystallform der so erstarrten Substanz ist dieselbe wie jene der aus Wasser oder Sublimation entstandenen Krystalle.

\section{Anti-Dimethylpimelinsäure,}

$\mathrm{COOH} . \mathrm{CH}\left(\mathrm{CH}_{3}\right) . \mathrm{CH}_{2} . \mathrm{CH}_{2} . \mathrm{CH}_{2} . \mathrm{CH}\left(\mathrm{CH}_{3}\right) . \mathrm{COOH}$.

Sclimelzpunkt $76^{0}-76,5^{0}$. Dargestellt von Kipping (I. c.).

Durch Verdunsten einer wässerigen Lösung der Anti-Säure uber Schwefelsäure wurden winzige, abgeflachte, durchsichtige Nadeln von etwa $0,5 \mathrm{~mm}$ Länge und $0,1 \mathrm{~mm}$ Durchmesser erhalten. Diese Kryställchen gehören dem rhombischen Systeme an und zeigen die Formen $m\{110\}$ und $r\{101\}$ (Fig. 8), die Flächen sind löcherig durch Anälzung und nur sehr schwierig messbar. Die Flächen von $m\{110\}$ sind stets bedeutend schmäler als jene von $r\{101\}$, welch letztere den Krystallen ein keilförmiges Aussehen verleihen und zu einer gerundeten messerartigen Schneide führen, durch welche sechs oder sieben Interferenzspectra zwischen gekreuzten Nicols sichtbar werden. Die Krystalle sind niemals doppelendig, da sie stets zu Rosetten von vier bis fünf Krystallen verwachsen sind, die Form $r\{101\}$ Fig. 8. wurde nur mit zwei Flăchen an den Krystallen beobachtet. Durch (101) erkennt man die wohlausgeprägte Interferenzfigur eines rhombischen Krystalles mit grossem Axenwinkel, welche wegen der Keilform der Flächen nicht ganz central ist. Axe $a$ ist spitze Bisectrix und die Ebene der optischen Axen ist parallel $b\{010\}$, Doppelbrechung positiv und mässig stark, ebenso die Dispersion.

Krystallsystem: Rhombisch.

$$
a: b: c=0,8207: 1: 2,862 .
$$

Beobachtete Formen: $m\{110\}, r\{101\}$. 
536 William J. Pope. Mitth. a. d. kryst. Labor. des »Central Technical College«. III.

$$
\begin{aligned}
& \text { Anzahl der } \\
& \text { Beobachlungen: Grenzen: Mittel: Berechnet: } \\
& m m=(110):(110) \quad 16 \quad 99^{0} 15^{\prime}-103^{0} \quad 8^{\prime} \quad 101^{0} 15^{\prime} \quad 104^{\prime} 45 \\
& m m=(110):(1 \pi 0) \quad 16 \quad 75 \quad 20-8137 \quad 7840 \quad- \\
& r r=(101):(\overline{101}) \quad 12 \quad 143 \quad 16-152 \quad 31 \quad 148 \quad 0 \quad- \\
& r r=(101):(10 T) \quad 8 \quad 3024-3719 \quad 3312 \quad 32 \quad 0
\end{aligned}
$$

Wie die vorher beschriebene Säure erstarrt auch diese auf dem Objectglase geschmolzen leicht von einem. Mittelpunkte aus, doch sind hierbei keine Bläschen zu bemerken, wie im vorhergehenden Falle. Die Krystalle sind identisch mit den aus Wasser erhaltenen; die spitze Mittellinie ist senkrecht zur Glasfläche, so dass das Axenbild an allen Stellen des Präparates zu sehen ist. Beim Verdampfen sublimirt die Säure in Rosetten oder undeutlich gebildeten Blättchen, welche stets die charakteristische Interferenzfigur zeigen. 